\title{
Enjoyment of Sexualization in Men and Women: Relationships with Ambivalent Sexism and Body Image Coping Strategies
}

\author{
Chiara Rollero $^{1}$ D $\cdot$ Carmen M Leon $^{2} \cdot$ Mara Martini $^{1} \cdot$ Norma De Piccoli $^{1}$
}

Accepted: 24 January 2022 / Published online: 23 February 2022

(c) The Author(s) 2022

\begin{abstract}
Enjoyment of sexualization (EoS) occurs when people find appearance-based sexual attention from others to be positive and rewarding. Scholars have not consistently established if enjoyment of being sexualized may be a form of sexual empowerment, promoting positive body acceptance, control, and self-care, or instead an endorsement of sexist ideologies, based on the idea that being sexually appealing to men is a component of the feminine gender role. In order to shed light on this, the present study aimed to analyze whether enjoyment of sexualization may foster adaptive or maladaptive coping strategies in case of threat to a positive body image. We also considered the role of ambivalent sexism in EoS processes, as well as that of self-esteem in body coping strategies. Considering the increasing sexualization of men in Western societies, the same relationships were tested in both men and women adults by means of a multigroup moderation analysis. Participants were 513 subjects $(32.3 \%$ men, $M=25.97$ years old) recruited from the Italian general population. Results showed that enjoyment of sexualization was positively related to both adaptive (i.e., positive rational acceptance) and maladaptive strategies (i.e., appearance fixing). Hostile sexism was positively linked to EoS in both men and women, whereas benevolent sexism was positively linked to EoS in the case of women and negatively in the case of men. Implications about the ambivalent nature of enjoyment of sexualization are discussed.
\end{abstract}

Keywords Ambivalent sexism · Body image coping strategies · Enjoyment of sexualization $\cdot$ Gender $\cdot$ Self-esteem

Chiara Rollero

chiara.rollero@unito.it

1 Department of Psychology, University of Turin, Via Verdi 10, 10124 Turin, Italy

2 School of Law, University of Castilla-La Mancha, Albacete, Spain 


\section{Introduction}

Sexualization relates to an emphasis on sexual appearance, physical beauty, and sexual appeal to other people (APA Task Force on the Sexualization of Girls, 2007) and alludes to the sexual activity and/or availability (Pacilli et al., 2019; Spaccatini, 2019). Although women are subject to increased sexualization relative to men, sexualized depictions of men are increasing (Daniel et al., 2014; Davids et al., 2020). This phenomenon is widely present in mass media, and the frequency and intensity of the sexualization of both women and men in advertisements, magazines, on television, and on the Internet have increased over the decades (Hatton \& Turner, 2011; Ward et al., 2016).

Sexualized portrayals of women and men are an issue of great relevance because behind them lies an important message about social expectations, values, and ideals. According to Ponterotto (2016), through sexualized depictions of women and men, society comes to accept a "norm" for bodily appearance alongside a particular set of characteristics, behaviors, and outcomes that are "normative" to that appearance. Gender socialization theories contribute to understanding the processes that explain that sexualized images of women and men influence thinking and form the basis for individuals' beliefs about how they should look and behave. The central premise of these theories is that children learn what it means to be either woman or man from prevailing cultural norms and are then reinforced for adhering to these norms (Papadopoulos, 2010). Furthermore, the exposure to such messages constrains individuals' conceptions of femininity and masculinity by putting appearance and physical attractiveness, as well as other characteristics associated to gender roles at the center of values. To illustrate the truth of this, research has found that the exposure to sexualized messages lead some women to believe that being sexually appealing to men is a component of the feminine gender role and their own identities, thus internalizing these social standards within their belief system (Bigler et al., 2019).

Although both women and men are sexualized, it is well-known that women are portrayed in a sexual manner more often than men (APA Task Force on the Sexualization of Girls, 2007). For this reason, research in this area has largely focused solely on women. To contribute to this field of research, the present study aims to analyze the relationship between the enjoyment of sexualization and the use of body image coping strategies in an Italian population sample composed by both women and men. At the same time, this study responds to the objective of examining the role of ambivalent sexism in enjoyment of sexualization processes, as well as that of self-esteem in body image coping strategies.

\section{Enjoyment of Sexualization, Sexual Empowerment, and Ambivalent Sexism}

The expression enjoyment of sexualization has currently a broad meaning and describes one's perception of sexualized attention. Although at the beginning this concept was only used regarding women (see Liss et al., 2011), recent research shows that men enjoy sexualization as much as women do (Visser et al., 2014; Ward et al., 
2016). Moreover, evidence suggests that women and men experience similar patterns when enjoy being sexualized. For instance, Visser et al. (2014) found that not only were mean levels of enjoyment of sexualization similar for women and men, but they also found that the patterns of relations between enjoyment of sexualization and body shame, self-esteem, and number of partners were not different for women and men.

Despite of the similarities, studies that have analyzed differences between women and men in the enjoyment of sexualization are scant. An important deficit since evidence suggests that factors associated to enjoy being sexualized could be different depending on gender (Fasoli et al., 2018). In this regard, women's enjoyment of sexualization has been related to a subjective sense of empowerment through the receipt of sexualized attention from men. Women are given the message that sexual empowerment presents an opportunity to feel "liberated" or "empowered" by the ability to exert control over their bodies and feel a sense of power in doing so (Baumgardner \& Richards, 2004; Bue \& Harrison, 2019; Levy, 2005). Empirical evidence shows that some women report gaining a sense of empowerment through being appreciated and admired sexually by men (Liss et al., 2011). Evidence also reveals that women who engage in self-sexualizing behaviors report them to be a manifestation of their sexual empowerment (Donaghue et al. 2011). However, some theorists argue that the act of presenting oneself as a sexual object makes self-sexualization a troubling phenomenon, thus suggesting that these behaviors are a form of oppression directed at controlling women (Choi \& DeLong, 2019; De Wilde et al., 2021; Gill, 2008). In this regard, some theorists have argued that the empowerment sold with sexualized behaviors is a far cry from actual feminist empowerment (De Wilde et al., 2019; Gill, 2008; Levy, 2005). Indeed, enjoyment of sexualization would be associated in women with more strongly subscribing to a sexist ideology, which perpetuates the position of women as inferior to and dependent on men and would lead women to happily accept their subordinate position (Levy, 2005; Saez et al., 2019).

Although in women the factors associated to the enjoyment of sexualization have been addressed, it is not clear whether enjoyment of sexualization increase empowerment in men. A priori, the relationship between men's enjoyment of sexualization and their sense of empowerment seems hard to comprehend. Moreover, the role of sexism in men's enjoyment of sexualization is still a question for future research. Nevertheless, some studies highlight the relevance of the adherence to traditional gender roles as one of the factors that might explain that men enjoy being sexualized (Smolak et al., 2014). Furthermore, a recent study has revealed that enjoyment of sexualization may stem from agreement with traditional sexist norms which assert that women should be considered attractive and pleasant, and men are considered to be dominant, strong, and powerful (Fasoli et al., 2018).

Sexism is a form of prejudice based on sex and is linked to beliefs around the fundamental nature of women and men and the roles they should play in society (European Institute for Gender Equality). According to the Ambivalent Sexism Theory (Glick \& Fiske, 1996), gender stereotypes comprise significant ambivalence, where positive feelings toward women coexist with a hostile dislike of them. Specifically, hostile sexism is an adversarial view of gender relations in which women are perceived as seeking to control men and usurping their power, whereas benevolent sexism idealizes women as pure creatures who ought to be protected, but it implies that 
women are weak and better suited for conventional gender roles (Glick \& Fiske, 1996, 2001). Empirical research has supported the link between ambivalent sexism and women's enjoyment of sexualization, showing that women who report greater enjoyment of sexualization also report greater endorsement of hostile and benevolent sexism (Riemer et al., 2020). For instance, Liss and colleagues (2011), in a sample composed of female college students, found that the EoS was uniquely predicted by both hostile and benevolent sexism, after controlling for surveillance and body shame. Similarly, Saez and colleagues (2019) examined the relationship between the enjoyment of sexualization and benevolent sexism, founding both variables were positively correlated.

If literature has clearly established the relationship between enjoyment of sexualization and ambivalent sexism in women, it remains unexplored whether similar patterns occur among men. Although research with man participants is considerably smaller, studies suggest that men enjoy sexualization just as much as women do (Visser et al., 2014; Ward et al., 2016). As Visser and colleagues (2014) argued, men's enjoyment of receiving sexual attention when displaying bare, shaved chests may not be vastly different from women's enjoyment of wearing revealing clothing. However, other scholars suggest that men's enjoyment of sexualization is not associated with how they conceive of themselves sexually (Barnett et al., 2018). While women who enjoy sexualization express a desire for men to appreciate their appearance (Liss et al., 2011), men's enjoyment of sexualization is more rooted in their desire for an ideal body image (Grieve \& Helmick, 2008; Oehlhof et al., 2009). Moreover, it has been found that for men, sexiness may not be merely a matter of body appearance but rather of power and dominance, aspects usually related to gender roles (see Smolak et al., 2014). Overall, this suggests that men's sexual image is not as easily impacted by women's view of them, as women's sexual image is by the opinion of men (Barnett et al., 2018). In other words, the EoS may occur in both genders, but it might be related to different constructs, due to the endorsement of traditional gender norms and roles. As a matter of fact, what makes men attractive may be not only how good-looking, strong, and fit they appear but also how powerful and conforming to gender roles they are (Fasoli et al., 2018). However, to our knowledge, the role of ambivalent sexism in the enjoyment of sexualization processes in samples composed by both women and men has not been investigated yet.

\section{Body Image, Coping Strategies, and Sexualization}

Body image comprises a person's feelings, attitudes, and perceptions about his or her physical appearance and incorporates body size estimation, evaluation of attractiveness, and emotions associated with size and shape (Grogan, 2010). Literature has shown that several psychological factors affect body image, such as self-esteem, internalization of societal body ideals, and aspects of gender-related social identity (Alleva, Martijn et al., 2015; Grogan, 2010; Slevec \& Tiggemann, 2011).

It appears reasonable that body image and self-esteem directly influence each other because if people do not like their bodies (or a part of their bodies), it is hard to feel good about their whole self. The reverse is also true: if individuals do not value 
their self, it is hard to notice the good things and give their bodies the respect they deserve. However, this relationship is mediated, among other factors, by mass media. Media influence leads people to internalize patterns of physical beauty, resulting in dissatisfaction with their own bodies when they are unable to match up to these patterns (Macedo et al., 2019). Experts have stated that healthy body image is more than simply tolerating what a person looks like. A healthy body image means that people truly accept and like the way they look and are not trying to change their bodies to fit the way they think they should look. In other words, a healthy body image means recognizing the individual qualities and strengths that make people feel good about their self beyond weight, shape or appearance, and resisting the pressure to strive for the myth of the "ideal body" that is seen in the media.

Although the link between body image and self-esteem is well-established, as well as the impact of mass media in a healthy body image, no studies have analyzed whether enjoyment of sexualization become a potential threat to a positive body image. Since enjoyment of sexualization is deeply related to the way people represent themselves sexualized and consider themselves attractive to others, it is considered relevant to analyze whether enjoyment of sexualization may foster adaptive or maladaptive coping strategies in case of threat to a positive body image. According to Cash and colleagues (2005), when specific contextual elements become a potential threat to a positive body image, individuals employ cognitive and behavioral strategies to cope with these distressing situations. The three main coping strategies are: avoidance, appearance fixing, and positive rational acceptance. Avoidance refers to the extent to which an individual will avert psychological discomfort through selfimposed ignorance of one's undesirable thoughts or feelings. Appearance fixing consists of the attempt to alter image with efforts to disguise, hide, camouflage, or alter the body area that the individual deems undesirable. Finally, positive rational acceptance comprises behavioral and mental strategies to pacify distress through positive self-care or rational self-talk about one's appearance (e.g., remain oneself the good qualities he/she has) (Cash et al., 2005). The use of avoidance and appearance fixing strategies is considered maladaptive because such strategies are related to disordered eating behaviors, lowered self-esteem, and lower quality of life (Bailey et al., 2016; Cash et al., 2005; Mancuso, 2016). On the contrary, positive rational acceptance is considered adaptive because it stems from higher self-esteem and is related to lower self-evaluative salience of physical appearance, as well as higher social support (Cash et al., 2005; Grower et al., in press).

\section{The Current Study}

The present study intends extending existing knowledge about enjoyment of sexualization. Specifically, the main aim is to investigate the relationship between EoS and the use of body image coping strategies (to our knowledge past research has not tested these relationships yet). Furthermore, we also consider the role of ambivalent sexism in EoS processes, as well as that of self-esteem, introduced as a control variable, in body image coping strategies. Finally, we test the same relationships among 
variables in both man and woman adults, extending past research that has tended to recruit only woman college samples (Bailey et al., 2016; Choma et al., 2009).

Based on previous research, concerning women, we hypothesized that:

(H1) Benevolent and hostile sexism would be positively related to EoS (Liss et al., 2011; Riemer et al., 2020; Saez et al., 2019);

(H2) if EoS may be intended as a form of empowerment, it would be positively related to positive acceptance (Bue \& Harrison, 2019; Choi \& DeLong, 2019; Liss et al., 2011; Saez et al., 2019); otherwise, it would be positively related to avoidance and appearance fixing (De Wilde et al., 2019; Erchull \& Liss, 2014; Liss et al., 2011; Visser et al., 2014);

(H3) self-esteem would be positively related to positive acceptance, and negatively related to avoidance and appearance fixing (Cash et al., 2005; Grower et al., in press).

As previous stated, literature on these issues has mainly focused on women. However, considering the increasing sexualization of men (Daniel et al., 2014; Davids et al., 2020; Parent \& Moradi, 2011; Visser et al., 2014), we may extend hypotheses $(\mathrm{H} 2)$ and $(\mathrm{H} 3)$ to men as well. Furthermore, in line with previous studies (Fasoli et al., 2018; Smolak et al., 2014), we may expect that male enjoyment of sexualization may not be merely a matter of body appearance but rather of power and dominance, rooted in the endorsement of traditional gender roles. For this reason, even hypothesis (H1) may be extended to men as well.

Finally, we decided to check for any mediational path in an explorative manner. To our knowledge, no previous study has tested the relationships we proposed in the model, so we had no specific hypothesis about mediational paths.

\section{Method}

\section{Participants and Procedure}

The study enrolled 513 heterosexual subjects $(32.3 \%$ man) recruited from the Italian general population. Their age ranged from 20 to $35(M=25.97, S D=3.87)$. All participants were Caucasian. One per cent had a basic education, $34.9 \%$ graduated from high school, and $64.1 \%$ had college degrees. Of the subjects, $64.6 \%$ were employed, $30.3 \%$ students, $4.7 \%$ unemployed, and $0.4 \%$ housewives.

Participants were recruited using snowball sampling begun with postings by the researchers and their students. They were told that the study was about the relationship between social attitudes and body related issues. The link from the posting took subjects to a secure, anonymous online questionnaire where they read an informed consent before beginning the study. Each participant was also informed that his/her participation was voluntary, and he/she could discontinue the study at any time. The questionnaire took approximately $20 \mathrm{~min}$ to complete. No compensation was given for participation. The Ethics Committee of the University of Turin approved the study protocol (protocol number 131,125/2020). 


\section{Measures}

The questionnaire included the following measures:

1. The short version of the Ambivalent Sexism Inventory (ASI; Glick \& Fiske, 1996; Rollero et al., 2014) including 6 items (Cronbach's $\alpha=0.86$ ) measuring Hostile Sexism toward women (e.g., "Women seek to gain power by getting control over men") and 6 items (Cronbach's $\alpha=0.84$ ) measuring Benevolent Sexism toward women (e.g., "Many women have a quality of purity that few men possess"). The items were rated on a 6-point point Likert-type scale ranging from "strongly disagree" (0) to "strongly agree" (5).

2. The Self-Esteem Scale (Rosenberg, 1965) including 10 items (Cronbach's $\alpha=0.89$ ) assessing personal self-esteem (e.g., "I feel that I am a person of worth, at least on an equal plane with others"). The items were rated on a 4-point scale ranging from "strongly disagree" (1) to "strongly agree" (4).

3. The Enjoyment of Sexualization Scale (Liss et al., 2011) including 8 items (Cronbach's $\alpha=0.88$ ) aimed at capturing the extent to which individuals seek to and enjoy emphasizing their sexiness (e.g., "I love to feel sexy", "I like showing off my body"). This measure was initially designed for women but was later shown to be valid for men (Visser et al., 2014). Participants rated their responses using a 6-point scale ranging from "strongly disagree" (1) to "strongly agree" (6).

4. The Body Image Coping Strategies Inventory (Cash et al., 2005; Rollero et al., 2017) used to assess coping strategies individuals use during an event that can negatively affect their body image, such as all the situations that may threaten their ability to feel okay about their looks. It included 9 items (Cronbach's $\alpha=0.79$ ) measuring Avoidance (e.g., "I withdraw and interact less with others"), 10 items (Cronbach's $\alpha=0.84$ ) measuring Appearance Fixing (e.g., "I spend extra time trying to fix what I don't like about my looks"), and 10 items (Cronbach's $\alpha=0.75$ ) measuring Positive Rational Acceptance (e.g., "I tell myself there are more important things than what I look like"). Participants responded to each item on a 4-point scale ranging from "definitely not me" $(0)$ to "definitely me" (3).

5. A brief list of socio-demographic items, including gender and sexual orientation (twenty-two subjects described themselves as not heterosexual and were discarded because sexual orientation may have a relevant and specific impact on the considered variables).

\section{Data Analyses}

First, we performed bivariate analyses using SPSS 27. We carried out T-tests to explore gender differences on the study variables and performed correlations between the scales separately by gender. Then, we tested the hypothesized relationships via structural equation modelling using AMOS 27. All variables in the model were treated as observed variables and the covariance matrices were analyzed using maximum likelihood estimation procedures. To investigate the moderating role of gender, gender invariance of paths was tested using multigroup moderation analysis. 
Finally, we check for mediational paths. Mediation analysis was conducted using bias-corrected bootstrapping (Preacher \& Hayes, 2008), computing the means of 500 estimated indirect effects by creating 500 bootstrap samples using random sampling with replacement. Significant mediation effects are detected if the $95 \%$ confidence intervals (CIs) of the indirect effects do not include zero.

\section{Results}

\section{Bivariate Analyses}

T-tests were performed to compared men and women scores across all the variables. As seen in Table 1, men outscored women on both Hostile and Benevolent Sexism. No gender difference emerged in relation to Self-esteem and Enjoyment of Sexualization. Concerning coping strategies in case of threatened body image, women scored higher than men on each dimension (i.e., Avoidance, Appearance Fixing, and Positive Rational Acceptance).

Table 2 shows the correlations between the scales. For both men and women sexist attitudes positively correlated with each other and with Enjoyment of Sexualization, which in turn was positively associated with Appearance Fixing. Self-esteem correlated negatively with Appearance Fixing and Avoidance, and only for women it was

Table 1 Differences between men and women: Mean scores and $t$ values

${ }^{* *} p<0.01 ; * p<0.05$

\begin{tabular}{|c|c|c|c|}
\hline & \multicolumn{2}{|c|}{ Mean scores } & \multirow[b]{2}{*}{$t$} \\
\hline & Women & Men & \\
\hline Hostile Sexism & 1.47 & 2.26 & $-7.06^{* *}$ \\
\hline Benevolent Sexism & 1.70 & 2.27 & $-4.86^{* *}$ \\
\hline Self-esteem & 3.06 & 3.16 & -1.84 \\
\hline Enjoyment of Sexualization & 3.63 & 3.68 & -1.09 \\
\hline Avoidance & 0.82 & 0.66 & $2.76^{*}$ \\
\hline Appearance Fixing & 1.50 & 1.28 & $3.75^{* *}$ \\
\hline Positive Rational Acceptance & 1.60 & 1.49 & $2.20^{*}$ \\
\hline
\end{tabular}

Table 2 Zero-order correlations between scales. Correlations for women are above the diagonal and correlations for men are below the diagonal

\begin{tabular}{llllllll}
\hline & 1 & 2 & 3 & 4 & 5 & 6 & 7 \\
\hline 1. Hostile Sexism & & $0.63^{* *}$ & 0.05 & $0.14^{* *}$ & -0.06 & 0.03 & 0.01 \\
2. Benevolent Sexism & $0.47^{* *}$ & & $0.11^{*}$ & $0.19^{* *}$ & $-0.13^{*}$ & 0.01 & 0.07 \\
3. Self-Esteem & -0.09 & 0.06 & & 0.03 & $-0.54^{* *}$ & $-0.23^{* *}$ & $0.17^{* *}$ \\
4. Enjoyment of Sexualization & $0.17^{*}$ & $0.17^{*}$ & 0.09 & & -0.01 & $0.44^{* *}$ & 0.05 \\
5. Avoidance & 0.02 & -0.05 & $-0.66^{* *}$ & 0.06 & & $0.32^{* *}$ & 0.01 \\
6. Appearance Fixing & 0.01 & 0.04 & $-0.31^{* *}$ & $0.43^{* *}$ & $0.30^{* *}$ & & 0.10 \\
7. Positive Rational Acceptance & 0.16 & -0.00 & -0.07 & 0.14 & $0.30^{* *}$ & $0.20^{*}$ & \\
\hline
\end{tabular}

$* * p<0.01, * p<0.05$ 
positively associated with Positive Rational Acceptance. In both groups Appearance Fixing and Avoidance were positively related, and only for men Positive Rational Acceptance was positively associated with Appearance Fixing and Avoidance.

\section{Testing the Hypothesized Model}

We tested the hypothesized paths between variables. The following steps were conducted: (a) an unconstrained multigroup model across gender was examined in which the same pattern of structural paths was tested without constraints across groups; and (b) a constrained multigroup model was examined, where structural paths were constrained to be equal across groups. As recommended (Hu \& Bentler, 1998; Keith, 2015), we tested the model fit by using different fit indexes to reduce the impact of their limits. Specifically, the following were used as criteria for acceptable model fit: 2, confirmatory fit index (CFI; Bentler, 1990), Tucker-Lewis index (TLI; Tucker \& Lewis, 1973), and root mean square error of approximation (RMSEA; Steiger, 1990). For CFI and TLI, values higher than 0.90 are considered satisfactory (Bentler, 1990). As for RMSEA values lower than 0.08 are considered satisfactory (Bentler, 1990). Furthermore, the Akaike information criterion (AIC) was used to compare the unconstrained and the constrained model. The absolute AIC value is irrelevant, but the models that generate lower AIC are better fitting (Keith, 2015).

Analysis of the unconstrained multigroup model indicated this model fit the data adequately: $\chi^{2}(18)=22.47$, n.s.; $\chi^{2} / \mathrm{gdl}=1.24 ; \mathrm{CFI}=0.99$; TLI $=0.99$; $\mathrm{RMSEA}=0.02$. The constrained multigroup model showed good fit indexes as well: $\chi^{2}(26)=41.29$, $p<0.05 ; \chi^{2} / \mathrm{gdl}=1.59 ; \mathrm{CFI}=0.98 ; \mathrm{TLI}=0.97$; RMSEA $=0.03$. However, AIC indicated that the unconstrained model $(\mathrm{AIC}=98.47)$ provided a significantly better fit to the data than the constrained one (AIC $=101.29)$. The significant Chi-square difference $\left(\Delta \chi^{2}=18.82, \Delta \mathrm{df}=8, p<0.05\right)$ revealed that one or more structural paths were different across gender. When this statistic is significant, the model with the smaller Chi-square is chosen (Yuan \& Bentler, 2006). Thus, we selected the baseline model as the final model.

The final model for men accounted for $27 \%$ of variance in Positive Acceptance, $26 \%$ of variance in Appearance Fixing, and 16\% of variance in Avoidance. The final model for women accounted for 30\% of variance in Positive Acceptance, $25 \%$ of variance in Appearance Fixing, and 29\% of variance in Avoidance.

Most of the paths were found to be significant in the hypothesized models across gender, with the exception of the path from Enjoyment of Sexualization and Avoidance, which was not significant in both genders. By testing structural invariance, we were able to see how each path coefficient differed by gender. First, in reference to Hypothesis 1, HS predicted Enjoyment of Sexualization in both groups ( $\operatorname{men} \beta=0.25$, $p<0.001$; women $\beta=0.11, p<0.05$ ), whereas BS was negatively related to Enjoyment of Sexualization in men $(\beta=-0.15, p<0.05)$ and positively in women $(\beta=0.12$, $p<0.05$ ). Second, concerning Hypothesis 2, for both men and women Enjoyment of Sexualization had a positive influence on Positive Acceptance (men $\beta=0.15$, $p<0.05$; women $\beta=0.14, p<0.01$ ) and Appearance Fixing (men $\beta=0.45, p<0.001$; women $\beta=0.48, p<0.001$ ). Finally, in reference to Hypothesis 3 , Self-Esteem was negatively related to Appearance Fixing (men $\beta=-0.30, p<0.001$; women $\beta=-0.26$, 
$p<0.001)$ and Avoidance (men $\beta=-0.61, p<0.001$; women $\beta=-0.48, p<0.001)$ in both groups, and positively related to Positive Acceptance in women only ( $\beta=0.13$, $p<0.01)$. Standardized coefficients for men and women are shown in the baseline model depicted in Fig. 1.

Multigroup moderation analysis was conducted using a sequential constraints approach in order to test whether paths were significantly variant by gender. Individual pathways were tested for invariance by successively constraining each path to be equal across groups to locate the variances in the model. Corresponding chi-square difference tests were then used to determine whether gender significantly moderated the paths. Results showed that gender moderated the relations between BS and Enjoyment of Sexualization, $\Delta \chi^{2}(1)=9.61, p<.01$, and Self-Esteem and Positive Acceptance, $\Delta \chi^{2}(1)=8.02, p<.01$.

Finally, the bootstrap analyses (500 resamples used) allowed to estimate the indirect effects. In both men and women Enjoyment of Sexualization was a significant partial mediator of the relation between HS and Positive Acceptance (men $\beta=0.04$; $95 \%$ CI 0.01 to $0.10 ; S E=0.02 ; p<0.05$; women $\beta=0.02 ; 95 \%$ CI 0.00 to 0.02 ; $S E=0.01 ; p<0.05$ ), and between HS and Appearance Fixing (men $\beta=0.11 ; 95 \% \mathrm{CI}$ 0.05 to $0.20 ; S E=0.03 ; p<0.01$; women $\beta=0.05 ; 95 \%$ CI 0.00 to 0.10 ; $S E=0.03$; $p<0.05)$. Enjoyment of Sexualization was a significant partial mediator also of the relation between BS and Positive Acceptance (men $\beta=-0.02 ; 95 \%$ CI -0.07 to $-0.00 ; S E=0.02 ; p<0.05$; women $\beta=0.02 ; 95 \%$ CI 0.00 to $0.05 ; S E=0.01 ; p<0.05$ ), and BS and Appearance Fixing (men $\beta=-0.07 ; 95 \% \mathrm{CI}-0.14$ to $-0.01 ; S E=0.03$; $p<0.05$; women $\beta=0.05 ; 95 \%$ CI 0.01 to $0.12 ; S E=0.03 ; p<0.05$ ).

\section{Discussion and Conclusion}

The aim of the present study was to investigate the relationship between the enjoyment of sexualization and the use of body image coping strategies (i.e., avoidance, appearance fixing, and positive rational acceptance) in order to clarify whether enjoying sexualization may represent a positive aspect within the body image domain. In addition, we examined in depth the role of ambivalent sexism in the enjoyment of sexualization processes. To do so, we proposed a model investigating the effect of ambivalent sexism on the enjoyment of sexualization, as well as that of self-esteem, introduced as a control variable, in body image coping strategies. To check whether patterns were similar across genders, we used a sample composed of both men and women. Findings from this study expand the literature in this field of research by analyzing whether enjoying being sexualized may foster adaptive or maladaptive body image coping strategies in both men and women. Furthermore, the present research advances in the study of gender differences within the enjoyment of sexualization, as well as the factors that are associated with it.

As seen from the results of descriptive statistics, men scored higher on both hostile and benevolent sexism when compared to women. These results are in line with previous research (Gluck et al., 2020; León \& Aizpurúa, 2020; Rollero \& Tartaglia, 2019 ) and suggest that the endorsement of sexist attitudes differs according to gender. As sexism relegates women to a place that is inferior to men, it seems reason- 

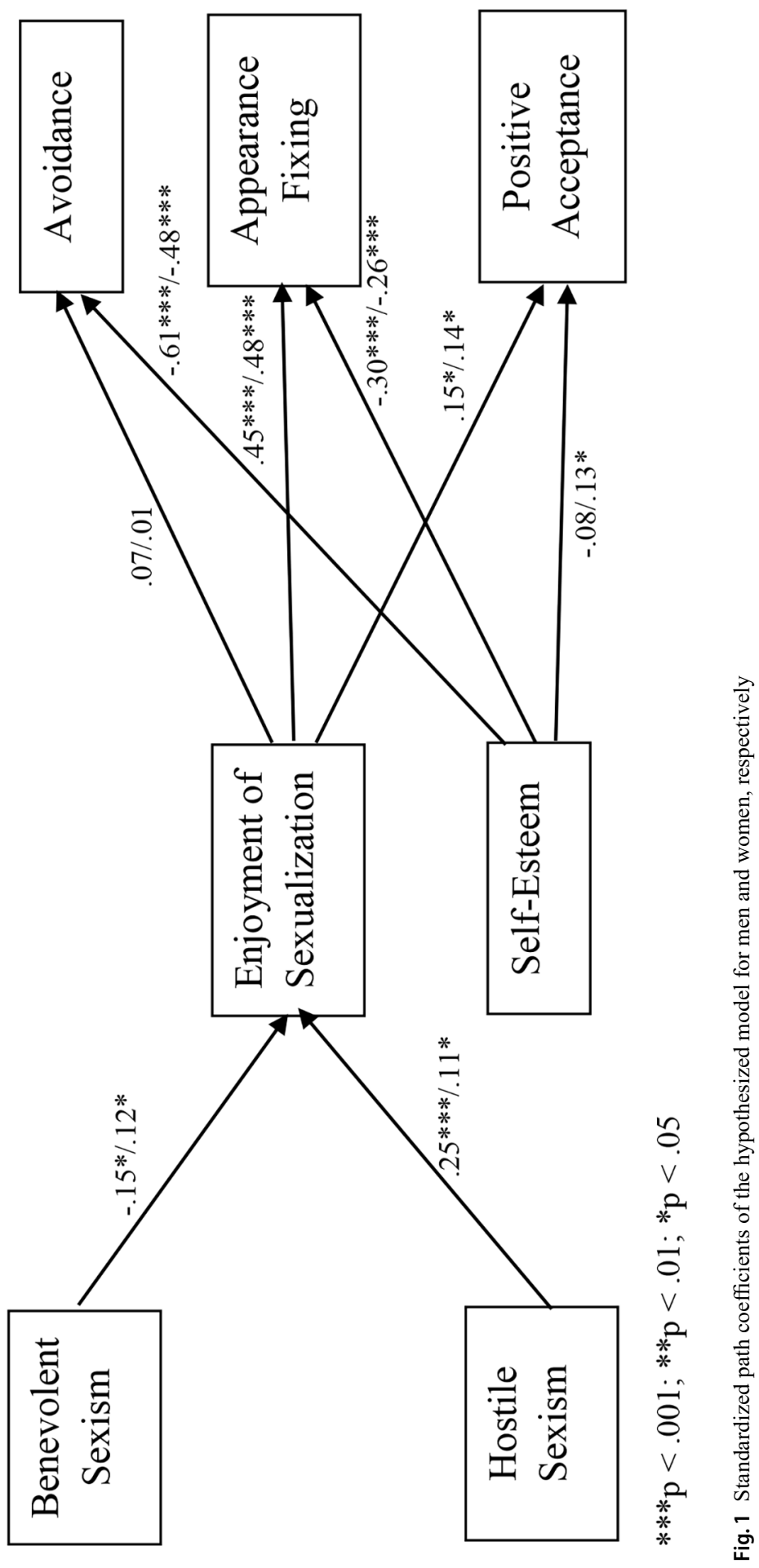
able that men are more ascribed to these attitudes. However, it should be noted that women themselves internalize and manifest sexist attitudes toward both themselves and other women, which is particularly manifested in the benevolent dimension due to its positive affective tone and its subtle and covert nature (Hammond \& Overall, 2017; León \& Aizpurúa, 2020). On the contrary, men and women showed similar levels of enjoyment of sexualization. This finding is in line with previous literature (Visser et al., 2014; Ward et al., 2016) and reveal that in Western societies (in which Italy is located) men enjoy sexualization as much as women do. However, similar to previous research (Cash et al., 2005), the results from this study showed that women use all three body image coping strategies (i.e., avoidance, appearance fixing, and positive rational acceptance) to a greater extent than men do. These results could be explained because women typically experience more body-image threats and are socialized more intensely to alter their appearance in the pursuit of cultural ideals when compared to men (Cash et al., 2005). Women generally evaluate and monitor themselves on a constant basis and as such internalize a certain kind of body shame due to a negative evaluation of their bodies because of a gap between what is ideal and what is real (Grippo \& Hill, 2008; Vandenbosch \& Eggermont, 2013). Their attempts at achieving these media ideals are intensified through self-sexualization behavior. Therefore, they are encouraged to alleviate their negative feelings and to boost their self-esteem through modifications in their appearance (Barzoki et al., 2016). Indeed, our results showed that women were especially prone to respond to body-image threats with appearance fixing behaviors.

Regarding the structural equations model, the findings provide partial support to our first hypothesis, showing that hostile sexism was positively related to the enjoyment of sexualization in both men and women. Benevolent sexism, however, was positively related to the enjoyment of sexualization in the case of women and negatively in the case of men. Although results regarding women have been extensively explained (see Liss et al., 2011; Riemer et al., 2020; Saez et al., 2019), they are novel regarding men. The existence of a link between hostile sexism and the enjoyment of sexualization in men suggests that traditional gender roles and expectations also affect the way that men enjoy their sexualization. For example, Amos and McCabe (2016) found that the largest predictor of men's perceived sexual attractiveness was adherence to the masculine gender norm, whereby men who felt they represent the "masculine" ideal perceived themselves as more sexually attractive. As Elkington (2021) has pointed out, "perhaps men consider masculine men more desirable to women, and as such, they have internalized the desire to be masculine in order to increase their mate value" (p. 12). Moreover, it has been found that notions of traditional masculinities that emphasize men's domination over women -socially and sexually-contribute to the stigmatization of men who fail to conform to these gendered expectations (Rollero, 2020). Which also might contribute to explain our results.

The finding showing that benevolent sexism had an opposite influence on men's and women's enjoyment of sexualization deserve further attention. Since benevolent sexism recognizes the women's dyadic power (i.e., the dependence of men on women for reproduction), the fact that the relationship between the enjoyment of sexualization and benevolent sexism was negative in men, might confirm that men's enjoyment of sexualization is more rooted in their desire for an ideal body image to feel more 
attractive to themselves, instead of a desire for being sexually appealing to women (Grieve \& Helmick, 2008; Oehlhof et al., 2009). Another possible interpretation lies in the characteristics of benevolent sexism. Benevolent sexism is an ideology that considers women as wonderful and pure creatures and allows men to maintain a positive self-image of paternalistic protectors and providers (Glick \& Fiske, 2001). Men who endorse such stereotypes adopt a traditional perspective on gender roles, which may be far from the sexualization processes of the man sex. Another possible explanation is addressed in the study conducted by Bosson et al. (2021), who found that the combination of high hostile sexism and low benevolent sexism related to men's low status in romantic domains. The authors explained that men with a low status in romantic domains view themselves as ugly and unappealing and believe they do not make attractive and valued romantic mates. Some of these men may include "incels", ${ }^{1}$ who typically believe their "unattractiveness blocks their access to female romantic and sexual partners". Moreover, the authors suggested that if low-mate value men doubt their protector and provider abilities (needed for benevolent sexism), they may find little motivation to embrace the chivalrous ideology of benevolent sexism that can offset high hostility and facilitate romantic interdependence.

Our results also revealed that the enjoyment of sexualization was positively related to positive rational acceptance (considered to be adaptive) and appearance fixing (considered maladaptive) in both men and women. These results partially support our second hypothesis and make difficult clearly indicate whether enjoying being sexualized represents a positive aspect of sexualization. Previous research shows that relying on positive rational acceptance represents a protective factor on individuals' well-being as it entails strategies emphasizing acceptance of the challenging event and positive self-care or rational self-talk about one's appearance (Cash et al., 2005). However, since appearance fixing is directed at altering appearance by covering, camouflaging, or correcting the perceived defect, appearance fixing coping has been found related to disordered eating attitudes (Choma et al., 2009). Overall, this suggest that there may exist some positive component to enjoyment of sexualization, which might counterbalance the negative component. Nevertheless, since the relationship between the enjoyment of sexualization and appearance fixing coping was stronger in both groups (men and women), the findings from this study seem to reinforce the negative impact the enjoyment of sexualization may have on healthrelated and behavioral coping outcomes. However, there might be some moderators (e.g., body image, body appreciation, attitudes toward sex) that can explain when and how enjoyment of sexualization can lead to adaptive versus maladaptive coping strategies. Future research should test the impact of these potential moderators, in order to extend our knowledge on the ambivalent nature of EoS. By linking these results with those found regarding sexism, our findings suggest that the enjoyment of sexualization is more related to conservative and traditional beliefs about gender norms than a form of gaining freedom and power through sexuality. Regarding women, the fact that enjoyment of sexualization was positively related to ambivalent sexism and appearance fixing coping strategies suggest that women who enjoy sexualization also

\footnotetext{
${ }^{1}$ Short for involuntary celibacy, "incel" refers to an internet subculture of men who blame women for the fact that they are not having sex.
} 
alter their appearance when their bodies do not conform to cultural ideals (Liss et al., 2011; Tiggemann \& Slater, 2013). In the case of men, the findings showed that they also alter their physical appearance in order to accomplish with the standardized masculine role (e.g., muscular bodies and shaved chests). This might reflect that men also follow some beauty rituals to fit on corresponding man attractiveness (e.g., going to the gym or taking supplements and anabolic steroids).

As far as our last hypothesis is concerned, as expected, the results showed that for both men and women, self-esteem was negatively related to avoidance and appearance fixing. These results are in line with previous research (Cash et al., 2005) and seem reasonable since avoidance and appearance fixing strategies are considered maladaptive. However, self-esteem was positively related to rational acceptance in the case of women only, yielding no significant results for men. This result highlights that self-esteem is more closely related to body image and physical appearance in women than in men. This finding may be related to the fact that men tend to show a stronger body appreciation compared to women and this may increase their ability to withstand body image threats (Sundgot-Borgen et al., 2021). In other words, men's self-esteem may discourage the use of maladaptive coping strategies, and at the same time may render unnecessary the use of adaptive mechanisms. Furthermore, self-esteem was included in the study as a control variable. However, it may also represent a potential moderator in the relationship between EoS and coping strategies. Future research on potential moderators could test also the role of self-esteem.

Although this study provides an important contribution to the existing literature, limitations should be noted. The first one refers to the narrow generalizability of the sample. Despite of this study is one of the few ones that used a general population sample composed by both men and women, data was collected by using a non-probability sampling. Another limitation is the socio-demographic profile of the sample, which was largely represented Caucasian and early adulthood participants (20-35 years old). For future studies, it may be important to include people from different ages and ethnicities. Additionally, it would have been worthy including measures to assess the endorsement of gender roles (and not only instruments measuring sexism). Finally, we used self-report measures, which may be affected by social desirability, especially considering coping strategies used in case of body image threat.

To summarize, our findings suggest that the dynamics surrounding the enjoyment of sexualization in men and women are complex. In line with Visser et al. (2014), in the current study, not only mean levels of enjoyment of sexualization were similar for men and women, but in our structural equations model, the patterns of relations between the enjoyment of sexualization, ambivalent sexism, and body image coping strategies were similar for men and women (except for the relationship between the enjoyment of sexualization and benevolent sexism, and self-esteem and positive rational acceptance). Taking findings all together, some of our results indicate that being sexualized might be a source of gaining freedom and power (e.g., the relationships with positive rational acceptance and self-esteem). On the other hand, some of the relationships among variables point to the fact that the enjoyment of sexualization is not actually empowering (e.g., the relationship with ambivalent sexism and fixing coping strategies). Whether the role of ambivalent sexism and other gender-related variables, such as the adherence to traditional gender roles, could explain differences 
in the patterns of enjoyment of sexualization among men and women is a question for future research.

\section{Practical Implications}

The topics of sexuality, gender, and the body are controversial in our current culture. The idea of sexual freedom emphasizes that people have the liberty to do whatever they want with their bodies. Regarding women, sexually liberal feminism propagates the idea of sexual freedom being an essential component of women's freedom. Paradoxically, research has shown that the act of presenting oneself as a sexual object makes self-sexualization a troubling phenomenon. In this regard, some studies state that enjoyment of sexualization is a form of oppression directed at controlling women instead of a form of empowerment (Choi \& DeLong, 2019; De Wilde et al., 2021). Along similar lines, it has been found that enjoyment of sexualization has also negative consequences for men (Visser et al., 2014).

Another important fact in the study of the enjoyment of sexualization is the increasing use of social networks and the troubling use made of them by younger boys and girls. As the majority of social networks have as the main purpose sharing images, these places are especially prone to self-sexualizing behaviors. A recent study carried out by the Royal Society for Public Health (2017) suggests that "young people who are heavy users of social media - spending more than two hours per day on social networking sites such as Facebook, Twitter or Instagram - are more likely to report poor mental health, including psychological distress (symptoms of anxiety and depression)" (p. 8). Because the image that people project on social networks structures relationships and turns into a measure of success, it is considered important to promote different strategies aimed at supporting early action programs with, especially, young men and women (Rollero et al., 2019). For example, it would be worth receiving notifications from the apps themselves warning of an excessive use. Moreover, it would be helpful that social networks warns when a photo is manipulated. It is expected that the strategies proposed empower people with the necessary knowledge and tools to protect them and promote their health and well-being when they enjoy sexualization.

\section{Declarations}

No funding was received for conducting this study.

The authors have no conflicts of interest to declare.

The Ethics Committee of the University of Turin, Italy, approved the study protocol.

The study was performed in accordance with the ethical standards as laid down in the 1964 Declaration of Helsinki and its later amendments.

Informed consent was obtained from all individual participants included in the study. 
Open Access This article is licensed under a Creative Commons Attribution 4.0 International License, which permits use, sharing, adaptation, distribution and reproduction in any medium or format, as long as you give appropriate credit to the original author(s) and the source, provide a link to the Creative Commons licence, and indicate if changes were made. The images or other third party material in this article are included in the article's Creative Commons licence, unless indicated otherwise in a credit line to the material. If material is not included in the article's Creative Commons licence and your intended use is not permitted by statutory regulation or exceeds the permitted use, you will need to obtain permission directly from the copyright holder. To view a copy of this licence, visit http:/creativecommons.org/ licenses/by/4.0/.

\section{References}

Alleva, J. M., Martijn, C., Van Breukelen, G. J. P., Jansen, A., \& Karos, K. (2015). Expand Your Horizon: A programme that improves body image and reduces self-objectification by training women to focus on body functionality. Body Image, 15, 81-89. doi: https://doi.org/10.1016/j.bodyim.2015.07.001

Amos, N., \& McCabe, M. (2016). Positive perceptions of genital appearance and feeling sexually attractive: Is it a matter of sexual esteem? Archives of Sexual Behavior, 45, 1249-1258. doi: https://doi. org/10.1007/s10508-015-0680-4

APA Task Force on the Sexualization of Girls (2007). Report of the APA Task Force on the Sexualization of Girls. American Psychological Association. www.apa.org/pi/wpo/sexualization.html

Bailey, K. A., Lamarch, L., Gammage, K. L., \& Sullivan, P. J. (2016). Self-objectification and the use of body image coping strategies: The role of shame in highly physically active women. The American Journal of Psychology, 129(1), 81-90. doi: https://doi.org/10.5406/amerjpsyc.129.1.0081

Barnett, M., Maciel, I., \& Gerner, M. (2018). Enjoyment of sexualization and feminism: Relationships with sexual self-schema and psychosexual health. Sexuality \& Culture, 22, 669-684. doi: https://doi. org/10.1007/s12119-018-9515-5

Barzoki, M. H., Mohtasham, L., Shahidi, M., \& Tavakol, M. (2016). Self-objectification and self-sexualization behavior within consumer culture. Applied Research in Quality of Life, 12(2), 425-438. doi: https://doi.org/10.1007/s11482-016-9468-5

Baumgardner, J., \& Richards, A. (2004). Feminism and femininity: Or how we learned to stop worrying and love the thong. In A. Harris (Ed.), All about the girl: Culture, power, and identity (pp. 59-67). Routledge

Bentler, P. M. (1990). Comparative fit indexes in structural models. Psychological Bulletin, 107, $238-246$. doi: https://doi.org/10.1037/0033-2909.107.2.238

Bigler, R. S., Tomasetto, C., \& McKenney, S. (2019). Sexualization and youth: Concepts, theories, and models. International Journal of Behavioral Development, 43, 530-540. doi: https://doi. org/10.1177/0165025419870611

Bosson, J. K., Rousis, G. J., \& Felig, R. N. (2021). Curvilinear sexism and its links to men's perceived mate value. Personality and Social Psychology Bulletin. doi: https://doi.org/10.1177/01461672211009726

Bue, A. C. C., \& Harrison, K. (2019). Empowerment sold separately: Two experiments examine the effects of ostensibly empowering beauty advertisements on women's empowerment and self-objectification. Sex Roles, 81(9), 627-642. doi: https://doi.org/10.1007/s11199-019-01020-4

Cash, T. F., Santos, M. T., \& Williams, E. F. (2005). Coping with body-image threats and challenges: Validation of the Body Image Coping Strategies Inventory. Journal of Psychosomatic Research, 58, 191-199. doi: https://doi.org/10.1016/j.jpsychores.2004.07.008

Choi, D., \& DeLong, M. (2019). Defining Woman Self Sexualization for the Twenty-First Century. Sexuality \& Culture, 23(4), 1350-1371. doi: https://doi.org/10.1007/s12119-019-09617-3

Choma, B. L., Shove, C., Busseri, M. A., Sadava, S. W., \& Hosker, A. (2009). Assessing the role of body image coping strategies as mediators or moderators of the links between self-objectification, body shame, and well-being. Sex Roles, 61(9-10), 699. doi: https://doi.org/10.1007/s11199-009-9666-9

Daniel, S., Bridges, S. K., \& Martens, M. P. (2014). The development and validation of the Man Assessment of Self-Objectification (MASO). Psychology of Men \& Masculinity, 15, 78-89. doi: https://doi. org/10.1037/a0031518

Davids, C. M., Watson, L. B., \& Gere, M. P. (2020). Objectification, masculinity, and muscularity: A test of objectification theory with heterosexual men. Sex Roles: A Journal of Research, 80(7-8), 443-457. doi: https://doi.org/10.1007/s11199-018-0940-6 
Donaghue, N., Kurz, T., \& Whitehead, K. (2011). Spinning the pole: A discursive analysis of the websites of recreational pole dancing studios. Feminism \& Psychology, 21(4), 443-457. doi: https://doi. org/10.1177/0959353511424367

De Wilde, M., Carrier, A., Casini, A., \& Demoulin, S. (2021). The Drawback of Sexual Empowerment: Perceiving Women as Emancipated but Still as Sexual Objects. Sex Roles, 84, 626-646. doi: https:// doi.org/10.1007/s11199-020-01192-4

De Wilde, M., Casini, A., Wollast, R., \& Demoulin, S. (2019). Sex is power belief and women's mental health: The mediating roles of self-objectification and sexual subjectivity. European Journal of Social Psychology, 50, 1017-1031. doi: https://doi.org/10.1002/ejsp.2643

Elkington, N. (2021). Validation of the enjoyment of sexualization scales in a midlife sample. [Master's thesis, The University of Guelph]. The Atrium

Erchull, M. J., \& Liss, M. (2014). The object of one's desire: How perceived sexual empowerment through objectification is related to sexual outcomes. Sexuality \& Culture, 18, 773-788. doi: https://doi. org/10.1007/s12119-013-9216-z

European Institute for Gender Equality. Glossary \& Thesaurus.https:/eige.europa.eu/thesaurus/overview

Fasoli, F., Durante, F., Mari, S., Zogmaister, C., \& Volpato, C. (2018). Shades of sexualization: When sexualization becomes sexual objectification. Sex Roles, 78, 338-351. doi: https://doi.org/10.1007/ s11199-017-0808-1

Gill, R. (2008). Empowerment/sexism: Figuring woman sexual agency in contemporary advertising. Feminism \& Psychology, 18, 35-60. doi: https://doi.org/10.1177/0959353507084950

Glick, P., \& Fiske, S. T. (1996). The Ambivalent Sexism Inventory: Differentiating hostile and benevolent sexism. Journal of Personality and Social Psychology, 70, 491-512. doi: https://doi. org/10.1037/0022-3514.70.3.491

Glick, P., \& Fiske, S. T. (2001). An ambivalent alliance: Hostile and benevolent sexism as complementary justifications for gender inequality. American Psychologist, 56(2), 109-118. doi: https://oi. org/10.1037/0003-066X.56.2.109

Gluck, M., Heesacker, M., \& Choi, H. D. (2020). How much of the dark triad is accounted for by sexism? Personality and Individual Differences, 154, 109728. doi: https://doi.org/10.1016/j.paid.2019.109728

Grieve, R., \& Helmick, A. (2008). The influence of men's self-objectification on the drive for muscularity: Self-esteem, body satisfaction and muscle dysmorphia. International Journal of Men's Health, 7 , 288. doi: https://doi.org/10.3149/jmh.0703.288

Grippo, K. P., \& Hill, M. S. (2008). Self-objectification, habitual body monitoring, and body dissatisfaction in older European American women: Exploring age and feminism as moderators. Body Image, 5(2), 173-182. doi: https://doi.org/10.1016/j.bodyim.2007.11.003

Grogan, S. (2010). Promoting Positive Body Image in Men and Women: Contemporary Issues and Future Directions. Sex Roles, 63(9-10), 757-765. doi: https://doi.org/10.1007/s11199-010-9894-z

Grower, P., Ward, L. M., \& Rowley, S. (in press). Beyond Objectification: Understanding the Correlates and Consequences of Sexualization for Black and White Adolescent Girls. Journal of Research on Adolescence. doi: https://doi.org/10.1111/jora.12598

Hammond, M. D., \& Overall, N. C. (2017). Dynamics within intimate relationships and the causes, consequences, and functions of sexist attitudes. Current Directions in Psychological Science, 26, 120-125. doi: https://doi.org/10.1177/0963721416686213

Hatton, E., \& Trautner, M. (2011). Equal opportunity objectification? The sexualization of men and women on the cover of rolling Stone. Sexuality \& Culture, 15(3), 256-278. doi: https://doi.org/10.1007/ s12119-011-9093-2

Hu, L. T., \& Bentler, P. M. (1998). Fit indices in covariance structure modeling: Sensitivity to underparameterized model misspecification. Psychological Methods, 3(4), 424-453. doi: https://doi. org/10.1037/1082-989X.3.4.424

Keith, T. Z. (2015). Multiple regression and beyond: An introduction to multiple regression and structural equation modeling (2nd ed.). New York, NY: Routledge

León, C. M., \& Aizpurúa, E. (2020). Do sexist attitudes persist in college students? An analysis of its prevalence, predictors, and gender differences. Educación XX1, 23(1), 275-296. doi: https://doi. org/10.5944/educXX1.23629

Levy, A. (2005). Woman chauvinist pigs: Women and the rise of raunch culture. Free Press

Liss, M., Erchull, M. J., \& Ramsey, L. R. (2011). Empowering or oppressing? Development and exploration of the enjoyment of sexualization scale. Personality and Social Psychology Bulletin, 37, 55-68. doi: https://doi.org/10.1177/0146167210386119 
Macedo, F. N., Macedo, N., da Costa, T. M., Pinheiro, R., de Castro, P. R., Machado, V. ... Alves, N. (2019). Influence of body dissatisfaction on the self-esteem Brazilian adolescents: A cross-sectional study. International Journal of Environmental Research and Public Health, 17(10), doi: https://doi. org/10.3390/ijerph17103536

Mancuso, S. G. (2016). Body image inflexibility mediates the relationship between body image evaluation and maladaptive body image coping strategies. Body Image, 16, 28-31. doi: https://doi.org/10.1016/j. bodyim.2015.10.003

Oehlhof, M. E. W., Musher-Eizenman, D. R., Neufeld, J. M., \& Hauser, J. C. (2009). Self-objectification and ideal body shape for men and women. Body Image, 6, 308-310. doi: https://doi.org/10.1016/j. bodyim.2009.05.002

Pacilli, M. G., Spaccatini, F., \& Tomasetto, C. (2019). Less human and help-worthy: Sexualization affects children's perceptions of and intentions toward bullied peers. International Journal of Behavioral Development, 43(6), 481-491. doi: https://doi.org/10.1177/0165025419873040

Papadopoulos, L. (2010). Sexualisation of young people. Review. http://girlsingangs.org/wordpress/wpcontent/uploads/2012/09/sexualisation-of-young-people-Papadopulous.pdf

Parent, M. C., \& Moradi, B. (2011). His biceps become him: A test of objectification theory's application to drive for muscularity and propensity for steroid use in college men. Journal of Counseling Psychology, 58, 246-256. doi: https://doi.org/10.1037/a0021398

Ponterotto, D. (2016). Resisting the male gaze: Feminist responses to the "normalization" of the female body in Western culture. Journal of International Women's Studies, 10(1), 133-151

Preacher, K. J., \& Hayes, A. F. (2008). Asymptotic and resampling strategies for assessing and comparing indirect effects in multiple mediator models. Behavior Research Methods, 40, 879-891. doi: https:// doi.org/10.3758/BRM.40.3.879

Riemer, A. R., Allen, J., Gullickson, M., \& Gervais, S. J. (2020). "You Can Catch More Flies with Honey than Vinegar": Objectification Valence Interacts with Women's Enjoyment of Sexualization to Influence Social Perceptions. Sex Roles, 83(11), 739-753. doi: https://doi.org/10.1007/ s11199-020-01143-z

Rollero, C. (2020). The social dimensions of intimate partner violence: A qualitative study with man perpetrators. Sexuality \& Culture, 24(3), 749-763. doi: https://doi.org/10.1007/s12119-019-09661-z

Rollero, C., Daniele, A., \& Tartaglia, S. (2019). Do men post and women view? The role of gender, personality and emotions in online social activity. Cyberpsychology: Journal of Psychosocial Research on Cyberspace, 13(1),), article 1. doi: https://doi.org/10.5817/CP2019-1-1

Rollero, C., Glick, P., \& Tartaglia, S. (2014). Psychometric properties of short versions of the Ambivalent Sexism Inventory and Ambivalence Toward Men Inventory. Testing, Psychometrics, Methodology in Applied Psychology, 21(2), 1-11. doi: https://doi.org/10.4473/TPM21.2.3

Rollero, C., \& Tartaglia, S. (2019). The effect of sexism and rape myths on victim blame. Sexuality \& Culture. doi: https://doi.org/10.1007/s12119-018-9549-8

Rollero, C., Tartaglia, S., \& De Piccoli, N. (2017). Psychometric properties of the Italian version of the Body Image Coping Strategies Inventory. Revista de Psicologia Social, 3, 293-305. doi: https://doi. org/10.1482/87886

Rosenberg, M. (1965). Society and the adolescent self-image. Princeton University Press

Royal Society for Public Health (2017). \#StatusOfMind Social median and young people's mental health and wellbeing. https://www.rsph.org.uk/static/uploaded/d125b27c-0b62-41c5-a2c0155a8887cd01. pdf

Saez, G., Valor-Segura, I., \& Expósito, F. (2019). Interpersonal sexual objectification experiences: Psychological and social well-being consequences for women. Journal of Interpersonal Violence, 34(4), 741-762. doi: https://doi.org/10.1177/0886260516645813

Slevec, J., \& Tiggemann, M. (2011). Media exposure, body dissatisfaction, and disordered eating in middle-aged women. Psychology of Women Quarterly, 35(4), 617-627. doi: https://doi. org/10.1177/0361684311420249

Smolak, L., Murnen, S. K., \& Myers, T. A. (2014). Sexualizing the self: What college women and men think about to be "sexy". Psychology of Women Quarterly, 38(3), 379-397. doi: https://doi. org/10.1177/0361684314524168

Spaccatini, F. (2019). Children's sexualization and appearance focus: A literature review. Psicologia sociale, 3/2019, 343-368. doi: https://doi.org/10.1482/94939

Steiger, J. H. (1990). Structural model evaluation and modification: An interval estimation approach. Multivariate Behavioral Research, 25, 173-180 
Sundgot-Borgen, C., Sundgot-Borgen, J., Bratland-Sanda, S., Kolle, E., Klugand, M., Svantorp-Tveiten, K. M. E., \& Fostervold, T. (2021). Body appreciation and body appearance pressure in Norwegian university students comparing exercise science students and other students. Public Health, 21, 532. doi: https://doi.org/10.1186/s12889-021-10550-0

Tiggemann, M., \& Slater, A. (2013). NetGirls: The Internet, Facebook, and body image concern in adolescent girls. International Journal of Eating Disorders, 46(6), 630-633. doi: https://doi.org/10.1002/ eat. 22141

Tucker, L. R., \& Lewis, C. (1973). A reliability coefficient for maximum likelihood factor analysis. Psychometrika, 38, 1-10. doi: https://doi.org/10.1007/BF02291170

Vandenbosch, L., \& Eggermont, S. (2013). The three-step process of self-objectification: Potential implications for adolescents' body consciousness during sexual activity. Body Image, 11(1), 77-80. doi: https://doi.org/10.1016/j.bodyim.2013.10.005

Visser, B. A., Sultani, F., Choma, B. L., \& Possebon, J. A. (2014). Enjoyment of sexualization: Is it different for men? Journal of Applied Social Psychology, 44(7), 495-504. doi: https://doi.org/10.1111/ jasp. 12241

Yuan, K. H., \& Bentler, P. M. (2006). Mean Comparison: Manifest Variable Versus Latent Variable. Psychometrika, 71(1), 139-159. doi: https://doi.org/10.1007/s11336-004-1181-x

Ward, L. M., Seabrook, R. C., Manago, A., \& Reed, L. (2016). Contributions of diverse media to selfsexualization among undergraduate women and men. Sex Roles, 74(1-2), 12-23. doi: https://doi. org/10.1007/s11199-015-0548-z

Publisher's Note Springer Nature remains neutral with regard to jurisdictional claims in published maps and institutional affiliations. 